\title{
Method to Determine the Applicability of Multi-Parameter Fusion Analysis
}

\author{
Xianliang He, Sanchao Liu*, Zehui Sun, Wenyu Ye, Jianwei Su, Haoyu Jiang, Ping Yang, Cadathur \\ Rajagopalan \\ Mindray, Shenzhen, China
}

\begin{abstract}
Alarm fatigue has long been a clinical problem. Multiparameter Fusion Analysis (MPA) is a technique often used to address this. However, it cannot be used in some cases such as in severe peripheral circulation disorder, cardiopulmonary resuscitation (CPR) etc.. In these cases, critical alarms may be erroneously suppressed if MPA is applied increasing clinical risk for subjects.

Electrocardiogram (ECG) and photoplethesmogram $(P P G)$ signals are normally synchronous. Based on this, our method evaluates the applicability of MPA as follows: Multiple physiological signals are first analyzed by highly reliable single-parameter analysis methods to obtain all the parameters from these as well as signal quality indices. Then, signal features of different channels for each cardiac cycle are grouped. Finally, group features are derived and used with an integrated decision tree to determine the applicability of MPA. Three databases were used to test algorithm performance. DB1 containing 200 clinical waveform records; DB2 with 100 clinical waveform records; DB3 comprising 30 cases recorded in cardiopulmonary resuscitation, each case spanning 2 hours. The recognition rates for the three databases were all greater than $90 \%$ indicating that the proposed method can effectively decide whether the considered physiological signals can be used for MPA.
\end{abstract}

\section{Introduction}

False alarms from patient monitors has been ranked as a top safety hazard in intensive care units ${ }^{[1]}$. In order to reduce the impact of alarm fatigue resulting from high rates of false alarms, MPA has been used to reduce false alarms, Qiao L. et $\mathrm{al}^{[2]}$ used a machine learning algorithm to fuse the features from the ECG, PPG and arterial blood pressure (ABP) and reduced more than 80 percent of false alarms for asystole and severe bradycardia. Wei $\mathrm{Z}$. et $\mathrm{al}^{[3]}$ developed an event feature for malignant arrhythmia detection, based on the waveform features of ABP and PPG, and showed that it could reduce false alarms by 50 percent. Multi-parameter fusion technology, if used appropriately, can greatly improve the accuracy of alarms.
However, it should be noted that there are some clinical situations where the underlying assumptions of MPA technology will not be valid. For example, in severe peripheral circulation disorder and CPR, the subject's cardiac rhythm often differs dramatically from a pulsatile rhythm or in some situations, different patients may be connected to the same monitor. Although the latter scenario is not commonly seen, we found, in post market clinical follow-ups that it is not uncommon in some regions that are short of monitors. Only when such issues are addressed properly can we use MPA technology without adding new risks.

This article introduces a method to address the issues described above. This method can be used as the first module of a MPA scheme to ensure the safety and effectiveness of data fusion.

\section{Methods}

A typical example of MPA technology is the joint analysis of ECG, PPG and IBP. During one cardiac cycle, the ECG records the heart's electrical activity, whereas the synchronized PPG and IBP record the pulsatile variation in the arterial blood resulting from the corresponding heart contraction - we refer to situations like this as MPA applicable situations in [4]. As shown in Fig.1, in MPA applicable situations, the temporal relationships between the metaphoric characteristics of these recordings are usually regular and consistent. On the contrary, when these signals are not synchronized, acquired from different people or from a person suffering circulatory collapse, the relationship will vary dramatically. We propose an algorithm that recognizes MPA applicable situations.

The proposed technique to determine the applicability of MPA mainly includes two parts as shown in Fig. 2: In the first part multiple physiological signals are analyzed by highly reliable single-parameter analysis methods to obtain the characteristics and parameters (heart rate, arrhythmia, ventricular fibrillation, asystole...) and signal quality index (SQI); In the second part, signal features of different channels are synchronized and grouped for each cardiac cycle. After that, the group feature sequence is derived and used for situation recognition. 


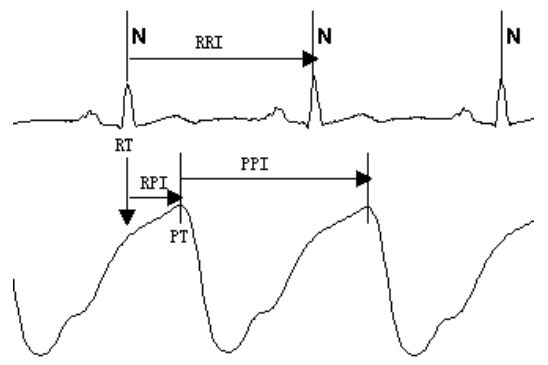

Figure 1. Features used in technique to determine MPA applicability. RRI: R-R interval, RT: R wave peak time, PPI: Pulse peak interval, PT: Peak time of pulse wave, RPI: $R$ peak-pulse peak interval. there is a high-risk ECG alarm such as ventricular fibrillation or asystole or a high-risk blood oxygen alarm such as extremely low oxygen saturation, or extremely low SQI caused by severe interference, or other situations like sensor disconnect MPA is left out.

The second part is feature sequence matching. In MPA applicable situations, the number of peaks in the ECG and PPG signals will be the same. In other situations, such as invalid heart ejection or severe signal interference, the number of $\mathrm{R}$ peaks could be different compared to peaks in the PPG, or the offset between the locations of these peaks varies dramatically, making it difficult to match them up. Therefore, it is very important to align the ECG signal features with those of the PPG signal in order to form a feature group sequence.

ECG and PPG are aligned as described below:

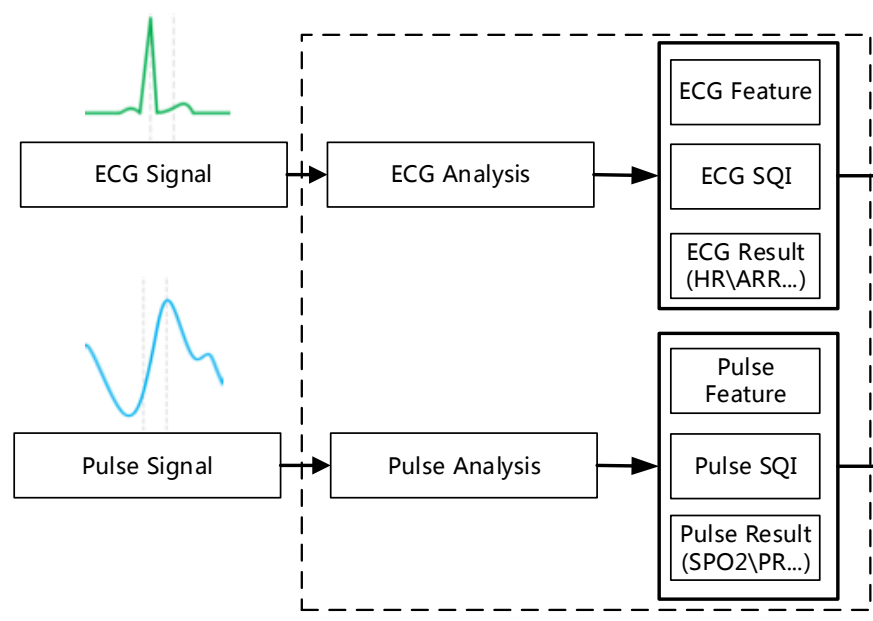

Figure 2. Technique to determine the applicability of MPA

\subsection{Single-parameter Signal Analysis}

After sampling and filtering, the multi-channel ECG signals are processed by the Mindray ECG Algorithm ${ }^{[5,6]}$ to accurately locate the QRS complexes and obtain heart rate, arrhythmias and SQI. PPG is also processed by our high performance algorithm to get SPO2, PPG SQI and to locate the pulse peaks ${ }^{[7,8,9]}$.

The results from the single parameter analysis are used as inputs to MPA, and after information fusion, MPA will further improve the accuracy of ECG and PPG parameters.

\subsection{Determination of MPA Applicability}

\subsubsection{Feature Sequence Matching}

The purpose of this module is to group ECG features and PPG features resulting from the same cardiac cycle by matching the ECG $\mathrm{R}$ peaks and PPG pulse peaks. It consists of two parts:

In the first step, abnormal feature sequences are checked and, if present, MPA is excluded. For example, if

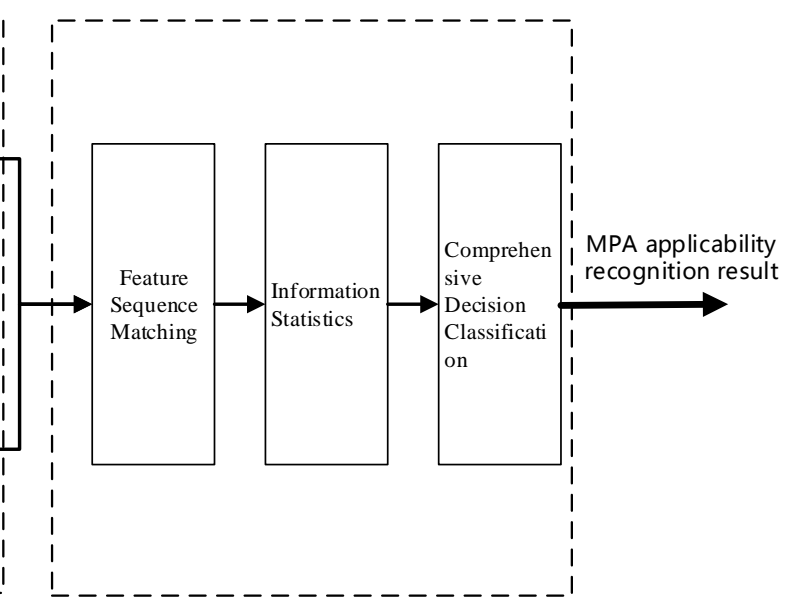

A pulse peak always occurs later in time than the corresponding ECG $\mathrm{R}$ peak. The time delay between a pulse peak and the corresponding $\mathrm{R}$ peak is called R-peak Pulse-peak Interval (RPI). Whenever an R peak is detected, a delay window is searched for the presence of a pulse peak. The width of this window is set to $100 \mathrm{~ms}$ to $200 \mathrm{~ms}$ before a new $\mathrm{R}$ peak is detected (details regarding the delay durations are discussed in [10].

If only one PPG peak is detected in the delay window, the pulse peak and the $\mathrm{R}$ peak will be grouped; if more than one pulse peak is detected (when signal amplitude is unusually low and interference is severe), a pulse peak will be selected based on the historical trend of RPI.

Three types of matches are possible between QRS wave and pulse wave:

1) Each QRS corresponds to a pulse wave, as shown in Figure 3, when the signal quality for both ECG and PPG is good. 


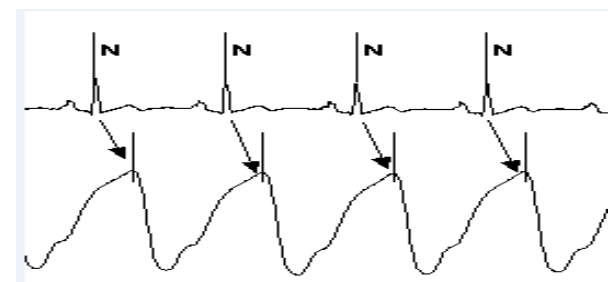

Figure 3. Each QRS has a matching pulse wave

2) Every QRS does not have a corresponding pulse wave as shown in Figure 4. Invalid ejection results in the absence of a pulse signal, so that the QRS complex has no corresponding pulse wave in the time window.

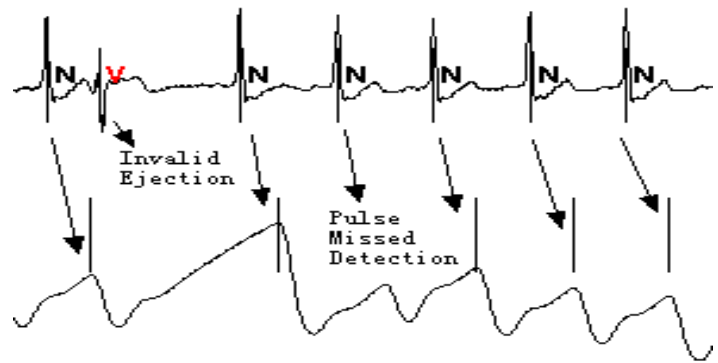

Figure 4. Every QRS does not have a matching pulse wave

3) A QRS wave corresponds to $\mathrm{N}$ pulse waves $(\mathrm{N}>1)$, as shown in Figure 5. Low signal amplitude and interference lead to missed QRS detection, which makes one QRS wave correspond to multiple pulse waves in the search window; False detection of pulse waves may also cause this.

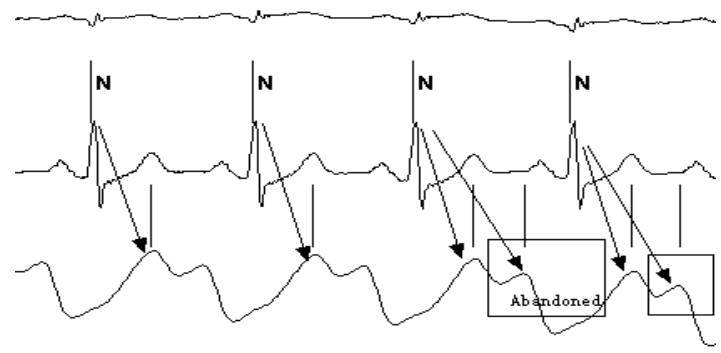

Figure 5. QRS wave with $\mathrm{N}$ pulse waves $(\mathrm{N}>1)$

\subsubsection{Information Statistics}

R-peak Pulse-peak Interval (RPI), R-peak Pulse-valley Interval (RVI) and their means and variances are calculated based on the grouped $\mathrm{R}$ peak and Pulse wave.

R-R intervals (RRI) are calculated for ECG while Peakpeak interval (PPI) and Valley-Valley Intervals (VVI) are calculated for PPG.

\subsubsection{Comprehensive Decision Classification}

MPA applicability is categorized into definitely applicable, applicable, not applicable and definitely not applicable, following a simple decision tree.

1) First, we compare the RRI from the ECG to the PPI and VVI from the PPG. If they vary greatly and the trends agree as in Fig. 6, MPA is definitely applicable. Otherwise,

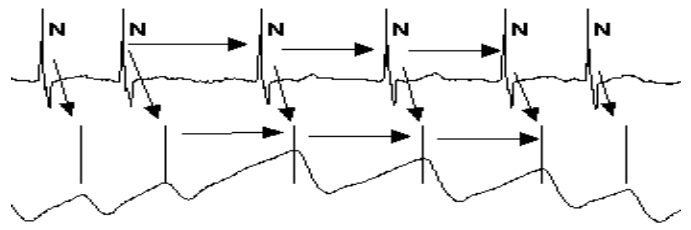

Figure 6. Joint feature type I

2) When premature ventricular contraction (PVC) is detected in the ECG, and the R-R intervals of the normal heart beats right before and after the PVC are close to the corresponding intervals of the pulse wave, as shown in Fig. 7, MPA is also definitely applicable. Otherwise,

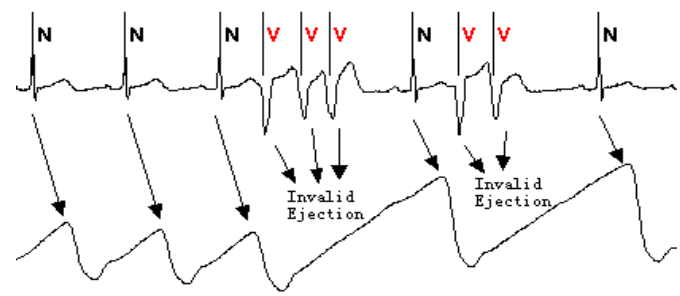

Figure 7. Joint feature type II

3) MPA applicability is decided according to the following rules:

- If the consistency ratio of RPI to RVI exceeds $80 \%$, MPA is applicable;

- If the number of effective RPI is less than 5, the previous judgment result is maintained.

- If the consistency ratio of RPI or RVI is less than $20 \%$, MPA is not applicable.

- If variances of RRI, PPI and VVI are all very small, but values have big difference, MPA is definitely not applicable.

\section{Results}

\subsection{Database Description}

We used three databases to evaluate the proposed method as shown in Table 1. DB1 contains 200 clinical recordings, each spanning 1 hour with heart rates ranging from 35 to 160 . DB2 was created using signals from DB1 to simulate MPA inapplicable situations. It contains 100 cases, with the ECG signals taken from the first 100 cases in DB1, paired with the PPG signals taken from the remaining 100 cases. DB3 contains 30 recordings collected in cardiopulmonary resuscitation, each spanning 2 hours. 


\subsection{Algorithm Evaluation}

We ran the algorithm on all the cases in DB1, DB2 and DB3. The algorithm evaluated the signals for MPA applicability every 15 seconds until a decision of definitely applicable or definitely not applicable is made and the evaluation process is only restarted when sensor off is detected (the sensors could later be possibly placed on a different subject). Following this strategy, a total of 1444, 775 , and 569 detections were made for DB1, DB2 and DB3 respectively. All the detections were reviewed retrospectively and marked as correct or false detections.

The results are listed in Table 1. DB1 was used to evaluate the identification of applicable cases. DB2 was employed to evaluate the identification of the not applicable (N/A) cases. Clinical cardiopulmonary resuscitation data in DB3 are mixed, with a majority being not applicable situations.

Table1. Algorithm results

\begin{tabular}{l|c|cc}
\hline \multirow{2}{*}{\multicolumn{2}{c|}{ Database }} & \multicolumn{2}{c}{ Evaluation results } \\
\cline { 3 - 4 } \multicolumn{2}{c}{ Total } & $\begin{array}{c}\text { Applicable } \\
\text { Total }(\%)\end{array}$ & $\begin{array}{c}\text { N/A } \\
\text { Total }(\%)\end{array}$ \\
\hline DB 1-applicable & 1444 & $\mathbf{1 3 5 1}(\mathbf{9 3 . 5 \%})$ & $93(6.5 \%)$ \\
DB 2-N/A & 775 & $43(5.6 \%)$ & $\mathbf{7 3 2}(\mathbf{9 4 . 4 \%})$ \\
DB 3-mixed & 569 & $62(10.9 \%)$ & $\mathbf{5 0 7}(\mathbf{8 9 . 1 \%})$ \\
\hline
\end{tabular}

\section{Discussion}

There are 93 "MPA not applicable" detections in DB1 whereas the truth is that all the cases in DB1 are MAP applicable. There are 3 possible reasons for such false detections. Firstly, the pulse peaks are not accurately located. Secondly, missed or falsely detected peaks appear in a sequence so long that it meets the decision condition for MPA not applicable. Lastly, the distributions of the group features have overlaps for applicable and not applicable situations. To alleviate false detection in those situations, we need to include more signal features and group features in the decision process. In practice, false MPA not applicable detections introduce no added clinical risk.

There are 43 MPA not applicable signal segments deemed MPA applicable in DB2. The main reason is that heart rate and pulse rate are close to each other while the difference of intervals varies very slowly. Fortunately, these false MPA applicable detections only lasted for a short period, and introduced little clinical risk.

DB3 contains CPR induced pulse waves interspersed with spontaneous circulations. The 62 MPA applicable detections were all spontaneous heart beats, and therefore are all true detections.

\section{Conclusions}

From the results above, it is seen that utilizing the consistency of the grouped metaphoric features to decide whether physiological signal can be used for MPA is effective.

Fusion of ECG and PPG is used in this paper as an example for MPA applicability recognition. Other vital signs such as fingertip PPG and invasive blood pressure can also be analysed together to evaluate the applicability of MPA for pulse waves. Generally, all the signals with high correlation can be considered for MPA applicability before use in multi-parameter fusion techniques.

\section{References}

[1] ECRI Institute. "Top 10 health technology hazards for 2014". Health Devices, vol. 42, no. 11, 2014.

[2] Qiao Li et al. "Signal quality and data fusion for false alarm reduction in the intensive care unit," J. Electrocardiol, vol. 45, no. 6, pp. 596-603, Mar. 2012.

[3] Wei Zong et al. "A practical algorithm to reduce false critica 1 ECG alarms using arterial blood pressure and/or photoplet hysmogram waveforms," Physiol.Meas, vol. 37, no. 8, pp. 1 355-1369, July. 2016.

[4] Ogedegbe G et al. "Principles and techniques of blood pressu re measurement," J. Cardiology Clinics, vol. 28, no. 4, pp. 5 71-586, Nov. 2016.

[5] Jianwei Su et al. "A four-lead real time arrhythmia analysis a lgorithm," Computing in Cardiology, vol. 44, no. , pp. 1-4, Sep. 2017.

[6] Zehui Sun et al. "Reducing ECG alarm fatigue based on SQI analysis.," Computing in Cardiology, vol. 41, no. , pp. 345 348, Sep. 2014.

[7] Juwon Lee et al. "Design of filter to reject artifact of pulse ox imetry," Computer Standards \& Interfaces, vol. 26, no. 3, pp. 241-249, May. 2004.

[8] Setsuo T.Peter W C.Edward A. "A non-invasive tissue reflec tance oximeter," Annals of Biomedical Engineering, vol. 8,no. 1, pp. 1-15, Jan. 1980.

[9] Yong-Sheng Yan et al. "Reduction of motion artifact in pulse oximetry by smoothed pseudo Wigner-Ville distribution," $J$. Neuroengineering And Rehabilitation, vol. 3, no. 2, pp. 1186-1743, Mar. 2005.

[10] Jorge Proenca et al. "Is Pulse Transit Time a good indicator of Bolld Pressure changes during short physical exercise in a young population?" 32nd Annual International Conference of the IEEE EMBS, vol. 2, no. 10, pp. 598-601, Aug. 2010.

Address for correspondence:

Sanchao Liu.

Mindray Building

Keji 12th Road South, High-Tech Industrial Park.Nanshan, Shenzhen, China

liusanchao@mindray.com 